\title{
Evidence for a Single-Spin Azimuthal Asymmetry in Semi-inclusive Pion Electroproduction
}

A. Airapetian, ${ }^{32}$ N. Akopov, ${ }^{32}$ M. Amarian, ${ }^{24,27,32}$ E. C. Aschenauer, ${ }^{13,14,6}$ H. Avakian, ${ }^{10}$ R. Avakian, ${ }^{32}$ A. Avetissian, ${ }^{32}$ E. Avetissian, ${ }^{10,32}$ B. Bains,${ }^{15}$ C. Baumgarten, ${ }^{22}$ M. Beckmann, ${ }^{12}$ S. Belostotski, ${ }^{25}$ J. E. Belz,${ }^{28,29}$ Th. Benisch, ${ }^{8}$

S. Bernreuther, ${ }^{8}$ N. Bianchi, ${ }^{10}$ J. Blouw ${ }^{24}$ H. Böttcher, ${ }^{6}$ A. Borissov, ${ }^{6,14}$ M. Bouwhuis, ${ }^{15}$ J. Brack,${ }^{4}$ S. Brauksiepe, ${ }^{12}$

B. Braun, ${ }^{22,8}$ B. Bray, ${ }^{3}$ St. Brons, ${ }^{6}$ W. Brückner, ${ }^{14}$ A. Brüll, ${ }^{14}$ E. E. W. Bruins, ${ }^{19}$ H. J. Bulten, ${ }^{18,24,31}$ G. P. Capitani, ${ }^{10}$

P. Carter, ${ }^{3}$ P. Chumney, ${ }^{23}$ E. Cisbani, ${ }^{27}$ G. R. Court, ${ }^{17}$ P. F. Dalpiaz, ${ }^{9}$ E. De Sanctis, ${ }^{10}$ D. De Schepper, ${ }^{19,2}$

E. Devitsin, ${ }^{21}$ P. K. A. de Witt Huberts,${ }^{24}$ P. Di Nezza, ${ }^{10}$ M. Düren, ${ }^{8}$ A. Dvoredsky, ${ }^{3}$ G. Elbakian, ${ }^{32}$ J. Ely, ${ }^{4}$ A. Fantoni, ${ }^{10}$ A. Fechtchenko, ${ }^{7}$ M. Ferstl, ${ }^{8}$ K. Fiedler ${ }^{8}$ B. W. Filippone, ${ }^{3}$ H. Fischer,${ }^{12}$ B. Fox, ${ }^{4}$ J. Franz, ${ }^{12}$ S. Frullani, ${ }^{27}$

M.-A. Funk, ${ }^{5}$ Y. Gärber, ${ }^{6}$ H. Gao, ${ }^{2,15,19}$ F. Garibaldi, ${ }^{27}$ G. Gavrilov,${ }^{25}$ P. Geiger, ${ }^{14}$ V. Gharibyan, ${ }^{32}$ A. Golendukhin, ${ }^{8,22,32}$ G. Graw, ${ }^{22}$ O. Grebeniouk, ${ }^{25}$ P.W. Green, ${ }^{1,29}$ L. G. Greeniaus, ${ }^{1,29}$ C. Grosshauser, ${ }^{8}$ M. Guidal, ${ }^{24}$ A. Gute, ${ }^{8}$

V. Gyurjyan, ${ }^{10}$ J. P. Haas, ${ }^{23}$ W. Haeberli, ${ }^{18}$ J.-O. Hansen, ${ }^{2}$ M. Hartig, ${ }^{29}$ D. Hasch,,${ }^{6,10}$ O. Häusser,${ }^{28,29, *}$ F. H. Heinsius, ${ }^{12}$ R. Henderson, ${ }^{29}$ M. Henoch, ${ }^{8}$ R. Hertenberger, ${ }^{22}$ Y. Holler, ${ }^{5}$ R. J. Holt, ${ }^{15}$ W. Hoprich, ${ }^{14}$ H. Ihssen, ${ }^{5,24}$ M. Iodice, ${ }^{27}$ A. Izotov, ${ }^{25}$ H. E. Jackson, ${ }^{2}$ A. Jgoun, ${ }^{25}$ R. Kaiser,${ }^{28,29,6}$ E. Kinney, ${ }^{4}$ A. Kisselev, ${ }^{25}$ P. Kitching, ${ }^{1}$ H. Kobayashi, ${ }^{30}$ N. Koch, ${ }^{8}$ K. Königsmann, ${ }^{12}$ M. Kolstein, ${ }^{24}$ H. Kolster, ${ }^{22}$ V. Korotkov, ${ }^{6}$ W. Korsch, ${ }^{3,16}$ V. Kozlov, ${ }^{21}$ L. H. Kramer, ${ }^{19,11}$ V. G. Krivokhijine, ${ }^{7}$ M. Kurisuno, ${ }^{30}$ G. Kyle,${ }^{23}$ W. Lachnit,${ }^{8}$ P. Lenisa, ${ }^{9}$ W. Lorenzon, ${ }^{20}$ N. C. R. Makins, ${ }^{2,15}$

F. K. Martens, ${ }^{1}$ J. W. Martin, ${ }^{19}$ F. Masoli, ${ }^{9}$ A. Mateos, ${ }^{19}$ M. McAndrew,${ }^{17}$ K. McIlhany, ${ }^{3,19}$ R. D. McKeown, ${ }^{3}$ F. Meissner, ${ }^{6}$ F. Menden, ${ }^{12,29}$ A. Metz, ${ }^{22}$ N. Meyners, ${ }^{5}$ O. Mikloukho, ${ }^{25}$ C. A. Miller ${ }^{1,29}$ M. A. Miller,${ }^{15}$ R. Milner, ${ }^{19}$ A. Most,${ }^{15,20}$ V. Muccifora, ${ }^{10}$ R. Mussa, ${ }^{9}$ A. Nagaitsev, ${ }^{7}$ Y. Naryshkin, ${ }^{25}$ A. M. Nathan, ${ }^{15}$ F. Neunreither, ${ }^{8}$ M. Niczyporuk, ${ }^{19}$ W.-D. Nowak, ${ }^{6}$ M. Nupieri, ${ }^{10}$ K. A. Oganessyan, ${ }^{10}$ T. G. O’Neill, ${ }^{2}$ R. Openshaw, ${ }^{29}$ J. Ouyang, ${ }^{29}$ B. R. Owen, ${ }^{15}$ V. Papavassiliou, ${ }^{23}$ S. F. Pate, ${ }^{19,23}$ M. Pitt, ${ }^{3}$ S. Potashov, ${ }^{21}$ D. H. Potterveld,${ }^{2}$ G. Rakness, ${ }^{4}$ A. Reali, ${ }^{9}$ R. Redwine, ${ }^{19}$ A. R. Reolon, ${ }^{10}$ R. Ristinen, ${ }^{4}$ K. Rith, ${ }^{8}$ P. Rossi, ${ }^{10}$ S. Rudnitsky, ${ }^{20}$ M. Ruh, ${ }^{12}$ D. Ryckbosch, ${ }^{13}$ Y. Sakemi,${ }^{30}$ I. Savin, ${ }^{7}$ C. Scarlett,${ }^{20}$ A. Schäfer, ${ }^{26}$ F. Schmidt,${ }^{8}$ H. Schmitt, ${ }^{12}$ G. Schnell, ${ }^{23}$ K. P. Schüler, ${ }^{5}$ A. Schwind, ${ }^{6}$ J. Seibert, ${ }^{12}$ T.-A. Shibata, ${ }^{30}$ K. Shibatani, ${ }^{30}$ T. Shin, ${ }^{19}$ V. Shutov, ${ }^{7}$ C. Simani, ${ }^{9}$ A. Simon, ${ }^{12}$ K. Sinram, ${ }^{5}$ P. Slavich,${ }^{9}{ }^{10}$ M. Spengos,${ }^{5}$ E. Steffens,${ }^{8}$ J. Stenger, ${ }^{8}$ J. Stewart, ${ }^{17}$ U. Stoesslein, ${ }^{6}$ M. Sutter, ${ }^{19}$ H. Tallini, ${ }^{17}$ S. Taroian, ${ }^{32}$ A. Terkulov, ${ }^{21}$ O. Teryaev, ${ }^{7,26}$ E. Thomas, ${ }^{10}$ B. Tipton, ${ }^{19}$ M. Tytgat, ${ }^{13}$ G. M. Urciuoli, ${ }^{27}$ J. F. J. van den Brand, ${ }^{24,31}$ G. van der Steenhoven, ${ }^{24}$ R. van de Vyver, ${ }^{13}$ J. J. van Hunen, ${ }^{24}$ M. C. Vetterli, ${ }^{28,29}$ V. Vikhrov, ${ }^{25}$ M. G. Vincter, ${ }^{29,1}$ J. Visser, ${ }^{24}$ E. Volk, ${ }^{14}$ W. Wander, ${ }^{8}$ J. Wendland, ${ }^{28}$ S.E. Williamson, ${ }^{15}$ T. Wise, ${ }^{18}$ K. Woller, ${ }^{5}$ S. Yoneyama, ${ }^{30}$ and H. Zohrabian ${ }^{32}$

(The HERMES Collaboration)

${ }^{1}$ Department of Physics, University of Alberta, Edmonton, Alberta, Canada T6G 2J1

${ }^{2}$ Physics Division, Argonne National Laboratory, Argonne, Illinois 60439-4843

${ }^{3}$ W. K. Kellogg Radiation Laboratory, California Institute of Technology, Pasadena, California 91125

${ }^{4}$ Nuclear Physics Laboratory, University of Colorado, Boulder, Colorado 80309-0446

${ }^{5}$ DESY, Deutsches Elektronen Synchrotron, 22603 Hamburg, Germany

${ }^{6}$ DESY Zeuthen, 15738 Zeuthen, Germany

${ }^{7}$ Joint Institute for Nuclear Research, 141980 Dubna, Russia

${ }^{8}$ Physikalisches Institut, Universität Erlangen-Nürnberg, 91058 Erlangen, Germany

${ }^{9}$ Istituto Nazionale di Fisica Nucleare, Sezione di Ferrara and Dipartimento di Fisica, Università di Ferrara, 44100 Ferrara, Italy

${ }^{10}$ Istituto Nazionale di Fisica Nucleare, Laboratori Nazionali di Frascati, 00044 Frascati, Italy

${ }^{11}$ Department of Physics, Florida International University, Miami, Florida 33199

${ }^{12}$ Fakultät für Physik, Universität Freiburg, 79104 Freiburg, Germany

${ }^{13}$ Department of Subatomic and Radiation Physics, University of Gent, 9000 Gent, Belgium

${ }^{14}$ Max-Planck-Institut für Kernphysik, 69029 Heidelberg, Germany

${ }^{15}$ Department of Physics, University of Illinois, Urbana, Illinois 61801

${ }^{16}$ Department of Physics and Astronomy, University of Kentucky, Lexington, Kentucky 40506

${ }^{17}$ Physics Department, University of Liverpool, Liverpool L69 7ZE, United Kingdom

${ }^{18}$ Department of Physics, University of Wisconsin-Madison, Madison, Wisconsin 53706

${ }^{19}$ Laboratory for Nuclear Science, Massachusetts Institute of Technology, Cambridge, Massachusetts 02139

${ }^{20}$ Randall Laboratory of Physics, University of Michigan, Ann Arbor, Michigan 48109-1120

${ }^{21}$ Lebedev Physical Institute, 117924 Moscow, Russia

${ }^{22}$ Sektion Physik, Universität München, 85748 Garching, Germany

${ }^{23}$ Department of Physics, New Mexico State University, Las Cruces, New Mexico 88003

${ }^{24}$ Nationaal Instituut voor Kernfysica en Hoge-Energiefysica (NIKHEF), 1009 DB Amsterdam, The Netherlands 


\author{
${ }^{25}$ Petersburg Nuclear Physics Institute, St. Petersburg, Gatchina, 188350 Russia \\ ${ }^{26}$ Institut für Theoretische Physik, Universität Regensburg, 93040 Regensburg, Germany \\ ${ }^{27}$ Istituto Nazionale di Fisica Nucleare, Sezione Sanità and Physics Laboratory, Istituto Superiore di Sanità, 00161 Roma, Italy \\ ${ }^{28}$ Department of Physics, Simon Fraser University, Burnaby, British Columbia, Canada V5A 1S6 \\ ${ }^{29}$ TRIUMF, Vancouver, British Columbia, Canada V6T 2 A3 \\ ${ }^{30}$ Department of Physics, Tokyo Institute of Technology, Tokyo 152-8551, Japan \\ ${ }^{31}$ Department of Physics and Astronomy, Vrije Universiteit, 1081 HV Amsterdam, The Netherlands \\ ${ }^{32}$ Yerevan Physics Institute, 375036, Yerevan, Armenia
}

(Received 28 October 1999)

\begin{abstract}
Single-spin asymmetries for semi-inclusive pion production in deep-inelastic scattering have been measured for the first time. A significant target-spin asymmetry of the distribution in the azimuthal angle $\phi$ of the pion relative to the lepton scattering plane was formed for $\pi^{+}$electroproduction on a longitudinally polarized hydrogen target. The corresponding analyzing power in the $\sin \phi$ moment of the cross section is $0.022 \pm 0.005 \pm 0.003$. This result can be interpreted as the effect of terms in the cross section involving chiral-odd spin distribution functions in combination with a chiral-odd fragmentation function that is sensitive to the transverse polarization of the fragmenting quark.
\end{abstract}

PACS numbers: 13.87.Fh, 13.60.-r, 13.88+e, 14.20.Dh

Polarized deep-inelastic lepton scattering has been the primary experimental basis for our present understanding of the spin structure of the nucleon. Inclusive and semiinclusive measurements with both beam and target polarized have been used to provide precise information on quark helicity-distribution functions. These quantities represent the distribution of quark spin in a longitudinally polarized nucleon, in a suitably Lorentz-boosted kinematic frame. Additional spin-distribution functions have been identified, but remain unmeasured. One of these is called transversity and corresponds to the distribution of transverse quark spin in a nucleon polarized transverse to its (infinite) momentum [1]. This and related distribution functions are predicted to be measurable via single-spin asymmetries, where only the beam or target are polarized, in certain lepton and hadron scattering experiments [2-6].

In simple models based on hadrons consisting of noninteracting collinear partons (quarks and gluons), single-spin asymmetries are expected to vanish. This follows from the conservation of parity, total angular momentum, and helicity of the individual partons. Correspondingly, in the language of perturbative quantum chromodynamics (QCD), single-spin asymmetries vanish at the "twist-2" level, i.e., when multiparton correlations and parton transverse momenta internal to hadrons are ignored. However, single-spin asymmetries have been observed in a few hadron-hadron scattering experiments [7]. In these measurements, a scattered hadron was detected with a momentum transverse to the beam direction in the range $P_{\perp} \simeq 1-2 \mathrm{GeV}$, which is not much larger than either the scale parameter of $\mathrm{QCD}\left(\Lambda_{\mathrm{QCD}} \sim 0.2 \mathrm{GeV}\right)$ or typical parton transverse momenta of a few hundred $\mathrm{MeV}$. Therefore these asymmetries may arise from noncollinear parton configurations or from multiparton correlations ("highertwist" effects), which are suppressed at large $P_{\perp}$ where perturbative QCD becomes effective.

Transversity and related spin-distribution functions are as yet unmeasured because their unusual chiral-odd structure implies that they are not directly observable in inclusive lepton-nucleon scattering experiments [1]. However, it has been suggested that the needed sensitivity can be provided by semi-inclusive production of pions with modest $P_{\perp}$ [2]. An observable single-spin dependence is predicted to appear in the dependence of the cross section on the angle between the spin axis of a transversely polarized target and the plane defined by the virtual photon momentum and the momentum of the pion (known as the Collins angle). Here the pion is produced from the struck quark in soft processes described by a fragmentation function having a chiral-odd structure like that of the spin-distribution functions of interest. This Collins fragmentation function describes how the probability for producing a pion depends on its direction with respect to the direction of transverse polarization of the struck quark. It also has a time-reversal-odd structure resulting from the final-state interactions in the fragmentation process, rather than from any fundamental violation of time-reversal invariance [8]. Such T-odd fragmentation (and distribution) functions can thus be considered as effective parametrizations of specific complex processes. There is preliminary evidence from $Z^{0} \rightarrow$ 2-jet decay [9] that the Collins fragmentation function has a substantial magnitude - of order $10 \%$ of the well-known chiral-even spin-independent one. If this can be confirmed, it could provide experimental sensitivity to the transverse polarization of scattered quarks in future experiments designed to make the first measurements of transversity.

In the case of semi-inclusive pion production in lepton scattering from a longitudinally polarized nucleon, chiralodd quark spin-distribution functions closely related to transversity can be manifest. In such experiments, the Collins angle becomes the azimuthal angle $\phi$ of the pion around the virtual photon direction, with respect to the lepton scattering plane. Recent theoretical studies [3,4] have shown how each chiral-odd spin-distribution function, coupled with the Collins fragmentation function, gives rise 
to a specific single-spin-dependent moment of the pion yield distribution in $\phi$.

The kinematics of the process are illustrated in Fig. 1. The relevant variables are the 4-momentum transfer squared $-Q^{2}=q^{2}=\left(k-k^{\prime}\right)^{2}$, the energy transfer $\nu=E-E^{\prime}$, the virtual photon fractional energy $y=$ $\nu / E$, the invariant mass of the photon-proton system $W=\sqrt{2 M \nu+M^{2}-Q^{2}}$, the Bjorken variable $x=Q^{2} /$ $2 M \nu$, and the pion fractional energy $z=E_{\pi} / \nu$. Here $k$ and $k^{\prime}$ are the 4-momenta and $E$ and $E^{\prime}$ are the laboratory energies of the incoming and outgoing leptons, respectively. $E_{\pi}$ is the pion laboratory energy and $M$ is the proton mass. The transverse momentum $\left(P_{\perp}\right)$ of the pion is defined with respect to the virtual photon direction in the initial photon-proton center-of-mass frame.

This Letter reports the first observation of a single-spin azimuthal asymmetry for semi-inclusive pion production in deep-inelastic scattering. The data were recorded during the 1996 and 1997 running periods of the HERMES experiment using both unpolarized and longitudinally nuclear-polarized hydrogen internal gas targets [10] in the $27.6 \mathrm{GeV}$ HERA polarized positron storage ring at DESY. Longitudinal beam polarization is obtained by using spin rotators [11] located upstream and downstream of the HERMES experiment. The scattered positrons and associated pions are detected by the HERMES spectrometer [12] in the polar angle range $0.04<\theta<0.22 \mathrm{rad}$. Positron and hadron identification is based on information from four detectors: a threshold gas Čerenkov counter, a transition-radiation detector, a preshower scintillator detector, and a lead-glass electromagnetic calorimeter. The particle identification provides an average positron identification efficiency of $99 \%$ with a hadron contamination that is less than $1 \%$.

The kinematic requirements on the scattered positron used in this analysis are $1<Q^{2}<15 \mathrm{GeV}^{2}, W>$ $2 \mathrm{GeV}, 0.023<x<0.4$, and $y<0.85$. Pions were identified in the energy range $4.5<E_{\pi}<13.5 \mathrm{GeV}$. Acceptance effects were minimized and exclusive production was suppressed by imposing the requirement $0.2<z<0.7$. The limit $P_{\perp}>50 \mathrm{MeV}$ was applied

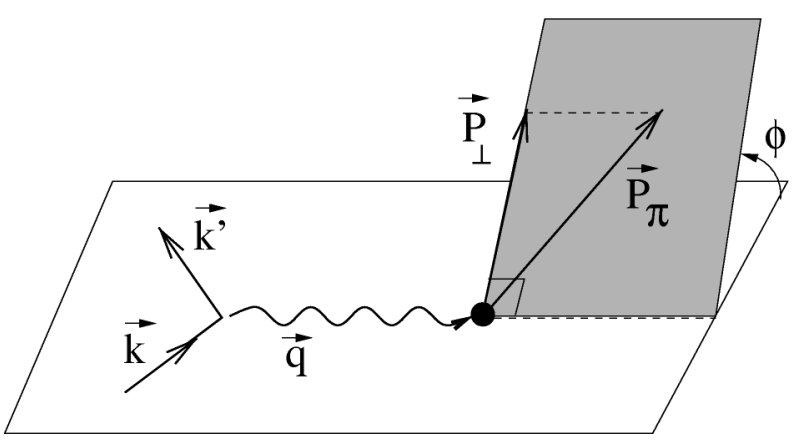

FIG. 1. Kinematic planes for pion production in semi-inclusive deep-inelastic scattering. to the pions to allow an accurate measurement of the angle $\phi$.

Measurements were performed with all combinations of beam and target helicities, giving the possibility of measuring single- and double-spin terms in the cross section. The average hydrogen target polarization in the 1996 and 1997 HERMES running periods was 0.86 with a fractional uncertainty of $5 \%$. The average beam polarization for the analyzed data was 0.55 with a fractional uncertainty of $3.4 \%$.

The various contributions to the $\phi$-dependent spin asymmetry are isolated by extracting moments of the cross section weighted by corresponding $\phi$-dependent functions. The analyzing powers for beam (target) longitudinal polarization are evaluated as

$$
A_{L U(U L)}^{W}=\frac{\frac{L^{\dagger}}{L_{P}^{\dagger}} \sum_{i=1}^{N^{\dagger}} W\left(\phi_{i}^{\dagger}\right)-\frac{L^{\downarrow}}{L_{P}^{\downarrow}} \sum_{i=1}^{N^{\downarrow}} W\left(\phi_{i}^{\downarrow}\right)}{\frac{1}{2}\left[N^{\dagger}+N^{\downarrow}\right]},
$$

where the $\uparrow / \downarrow$ denotes positive/negative helicity of the beam (target). Each summation is over the number $N^{\dagger / \downarrow}$ of selected events involving a detected pion for each beam (target) spin state corresponding to the dead-time corrected luminosities $L^{\dagger / \downarrow}$ and $L_{P}^{\uparrow / \downarrow}$, the latter being averaged with the magnitude of the beam (target) polarization. All of these quantities are effectively averaged over the two target (beam) helicity states to arrive at single-spin asymmetries. The weighting functions $W(\phi)=\sin \phi$ and $W(\phi)=\sin 2 \phi$ are expected to provide sensitivity to the Collins fragmentation function discussed above, in combination with different spin-distribution functions [3,4]. Analyzing powers were extracted by integrating over the spectrometer acceptance in the kinematic variables $y$ and $z$. Corrections were applied for the effects of the spectrometer acceptance, based on a Monte Carlo simulation.

The values of $A_{U L}^{\sin \phi}, A_{U L}^{\sin 2 \phi}$, and $A_{L U}^{\sin \phi}$ extracted from the data according to Eq. (1) and averaged over $x$ and $P_{\perp}$ are given in Table I. For both $\pi^{+}$and $\pi^{-}$the beam-related analyzing powers $A_{L U}^{\sin \phi}$ are consistent with zero. This is in agreement with the small contributions to $A_{L U}^{\sin \phi}$ predicted to arise from higher-twist and $O\left(\alpha_{S}^{2}\right)$ QCD effects [13,14]. The target-related term $A_{U L}^{\sin 2 \phi}$ is also consistent with zero within errors, both for $\pi^{+}$and $\pi^{-}$.

The other target-related analyzing power $A_{U L}^{\sin \phi}$ is consistent with zero for $\pi^{-}$, while it is significantly different from zero for $\pi^{+}$. The appearance of such an

TABLE I. Target- and beam-related analyzing powers, averaged over $x$ and $P_{\perp}$, for the azimuthal $\sin \phi$ and $\sin 2 \phi$ moments of the pion production cross section in deep-inelastic scattering.

\begin{tabular}{crc}
\hline \hline & \multicolumn{1}{c}{$\pi^{+}$} & $\pi^{-}$ \\
\hline$A_{U L}^{\sin \phi}$ & $0.022 \pm 0.005 \pm 0.003$ & $-0.002 \pm 0.006 \pm 0.004$ \\
$A_{U L}^{\sin 2 \phi}$ & $-0.002 \pm 0.005 \pm 0.010$ & $-0.005 \pm 0.006 \pm 0.005$ \\
$A_{L U}^{\sin \phi}$ & $-0.005 \pm 0.008 \pm 0.004$ & $-0.007 \pm 0.010 \pm 0.004$ \\
\hline \hline
\end{tabular}


asymmetry suggests the influence of the Collins T-odd fragmentation function, in combination with one or more chiral-odd spin-distribution functions. The large difference between the $\pi^{+}$and $\pi^{-}$asymmetries can be interpreted in the Quark Parton model to provide information about the flavor dependence of the chiral-odd spin-dependent fragmentation functions - i.e., the degree of enhancement of the "favored" Collins function describing fragmentation of a quark to a hadron containing that quark's flavor. The asymmetry for $\pi^{+}$is dominated by scattering from up quarks because the up quark is more abundant in the proton and has a higher charge than the down quark. On the other hand, the $\pi^{-}$asymmetry is more complicated as it receives significant contributions from both up and down quarks. These contributions probably tend to cancel because the up and down quarks can be expected to have opposite transverse polarizations, in analogy with the longitudinal case. Under such assumptions, it can easily be shown that the upper limit of about $\frac{1}{2}$ given by the present data for the ratio $A_{U L}^{\sin \phi}\left(\pi^{-}\right) / A_{U L}^{\sin \phi}\left(\pi^{+}\right)$indicates that the flavor dependence of the chiral-odd fragmentation functions is at least as strong as that of the chiral-even (unpolarized) functions. Recent theoretical work has shown that such a result is not unexpected [15].

In Table II the $A_{U L}^{\sin \phi}$ and $A_{U L}^{\sin 2 \phi}$ analyzing powers are given for $\pi^{+}$and $\pi^{-}$at the measured $\langle x\rangle$ and $\left\langle Q^{2}\right\rangle$ values. In addition, in Fig. 2, the $A_{U L}^{\sin \phi}$ and $A_{U L}^{\sin 2 \phi}$ values obtained for $\pi^{+}$are shown as a function of $x$, after averaging over $P_{\perp}$. At higher energies, the analyzing power for the $\sin \phi$ moment that is subleading order in $1 / Q$ is expected to be suppressed by the factor of $P_{\perp} / Q[3,4]$ with respect to the leading-order $\sin 2 \phi$ moment. In the HERMES kinematics, which covers a range of relatively low $Q^{2}$ and moderate $P_{\perp}$, the ratio of $A_{U L}^{\sin 2 \Phi}$ to $A_{U L}^{\sin \phi}$ analyzing powers is predicted to be small in the valence region [16]. This is in agreement with a simple estimate of that ratio in the real photon limit [17]. The present data are consistent with these theoretical expectations, neglecting the contribution to the $\sin \phi$ moment from transversity itself arising from the small component of the target-spin transverse to the virtual photon direction. Also, the apparent increase of $A_{U L}^{\sin \phi}$ with increasing $x$ suggests that the sea contribution does not dominate the effect, in agreement with existing interpretations of single-spin asymmetries as being associated with valence quark contributions $[18,19]$.

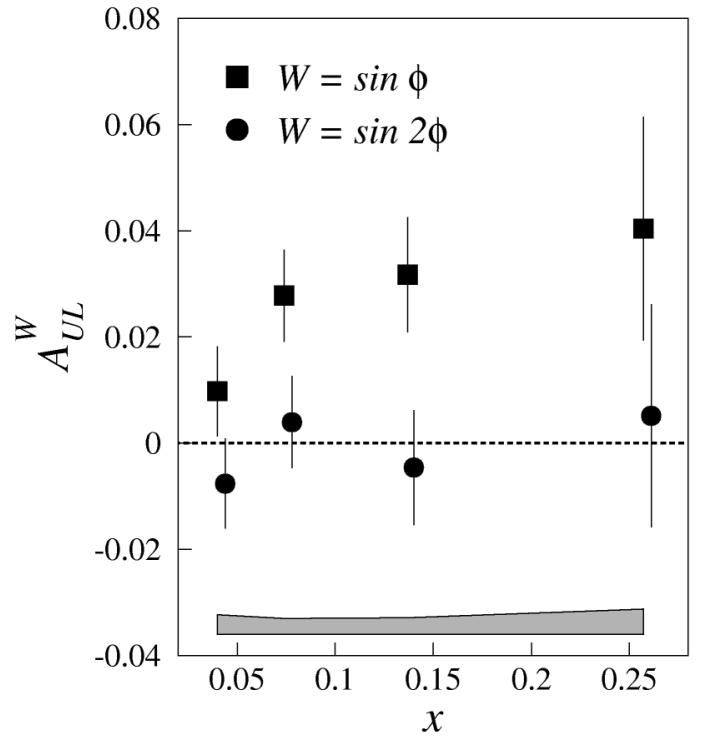

FIG. 2. Target-spin analyzing powers for $\pi^{+}: A_{U L}^{\sin \phi}$ (squares) and $A_{U L}^{\sin 2 \phi}$ (circles) as a function of Bjorken $x$. Error bars show the statistical uncertainty and the band represents the systematic uncertainties for $A_{U L}^{\sin \phi}$. As shown in Table II, $\left\langle Q^{2}\right\rangle$ varies with $x$.

In Fig. 3, $A_{U L}^{\sin \phi}$ averaged over $x$ is plotted for $\pi^{+}$and $\pi^{-}$as a function of transverse momentum. The mean $\langle Q\rangle$ is about $1.55 \mathrm{GeV}$ for all bins. There is an indication that $A_{U L}^{\sin \phi}$ for $\pi^{+}$increases as $P_{\perp}$ increases up to $\sim 0.8 \mathrm{GeV}$. This behavior can be related to the dominant role of the intrinsic quark transverse momentum when $P_{\perp}$ remains below a typical hadronic mass $(\sim 1 \mathrm{GeV})$. On this basis, the use of Gaussian transverse momentum parametrizations for distribution and fragmentation functions results in a behavior of $A_{U L}^{\sin \phi}$ that is proportional to $P_{\perp}$, at least for the moderate range of $P_{\perp}[3,4,20]$.

The main contributions to the systematic uncertainties are those from the target and beam polarizations, from smearing due to detector resolution, and from a false spin asymmetry induced by the spectrometer acceptance. Uncertainties in the acceptance corrections based on Monte Carlo calculations dominate the systematic uncertainties at small $x$ and decrease with increasing $x$. At the average values of $y$ of about 0.5 , radiative effects are expected to be small and independent of the pion charge; these effects on the unpolarized cross section were evaluated and were indeed found to be negligible [21].

TABLE II. Target-related analyzing powers averaged over $P_{\perp}$, for the azimuthal $\sin \phi$ and $\sin 2 \phi$ moment of the $\pi^{+}$and $\pi^{-}$ production cross section in deep-inelastic scattering as a function of $x$.

\begin{tabular}{ccccrr}
\hline \hline & \multicolumn{1}{c}{$\left\langle Q^{2}\right\rangle$} & $\pi^{+}$ & \multicolumn{1}{c}{${ }^{\sin \phi}$} & $\pi^{-}$ \\
$\langle x\rangle$ & $\left(\mathrm{GeV}^{2}\right)$ & $A_{U L}^{\sin \phi}$ & \multicolumn{1}{c}{$A_{U L}^{\sin }$} & \multicolumn{1}{c}{$A_{U L}^{\sin 2 \phi}$} \\
\hline 0.040 & 1.4 & $0.010 \pm 0.008 \pm 0.004$ & $-0.008 \pm 0.008 \pm 0.011$ & $-0.004 \pm 0.010 \pm 0.004$ & $0.002 \pm 0.010 \pm 0.008$ \\
0.074 & 2.2 & $0.028 \pm 0.009 \pm 0.003$ & $0.007 \pm 0.009 \pm 0.012$ & $-0.004 \pm 0.010 \pm 0.003$ & $-0.008 \pm 0.010 \pm 0.010$ \\
0.137 & 3.7 & $0.032 \pm 0.011 \pm 0.003$ & $-0.005 \pm 0.011 \pm 0.009$ & $0.012 \pm 0.013 \pm 0.003$ & $-0.007 \pm 0.013 \pm 0.007$ \\
0.257 & 6.4 & $0.041 \pm 0.021 \pm 0.005$ & $0.005 \pm 0.021 \pm 0.009$ & $-0.025 \pm 0.028 \pm 0.005$ & $-0.028 \pm 0.028 \pm 0.008$ \\
\hline \hline
\end{tabular}




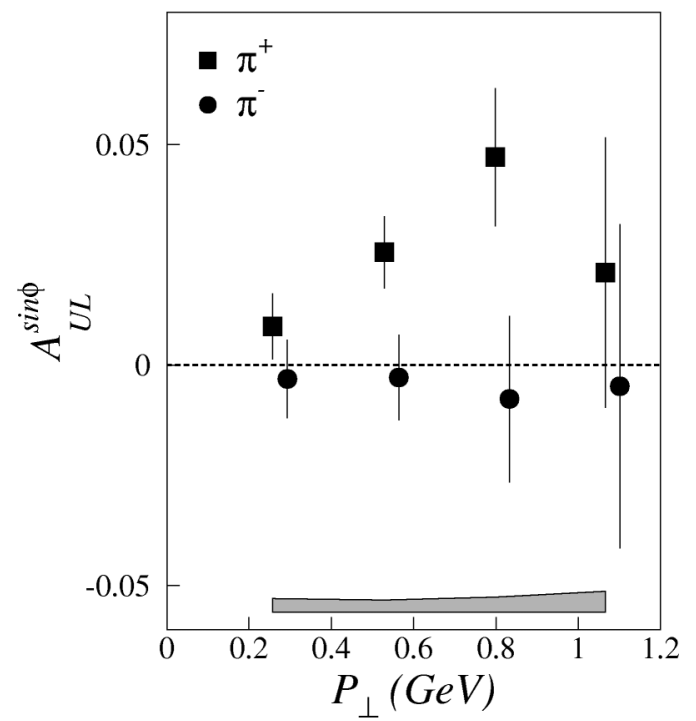

FIG. 3. Target-spin analyzing powers in the $\sin \phi$ moment as a function of transverse momentum, for $\pi^{+}$(squares) and $\pi^{-}$ (circles). Error bars show the statistical uncertainties and the band represents the systematic uncertainties.

In summary, single-spin azimuthal asymmetries of pions produced in deep-inelastic scattering of polarized positrons from a longitudinally polarized hydrogen target have been measured. The analyzing power involving the $\sin \phi$ moment of the cross section is found to be significant for $\pi^{+}$ production with unpolarized (spin-averaged) positrons on a longitudinally polarized hydrogen target, while for $\pi^{-}$it is found to be consistent with zero. In addition, the analyzing powers involving the $\sin 2 \phi$ moments of both $\pi^{+}$and $\pi^{-}$are consistent with zero. The $\sin \phi$ target-related analyzing power for $\pi^{+}$, averaged over the full acceptance, is found to be $0.022 \pm 0.005 \pm 0.003$, and there are indications that this analyzing power increases with increasing $x$, and also with $P_{\perp}$ up to $\sim 0.8 \mathrm{GeV}$. The appearance of this single-spin asymmetry can be interpreted as an effect of chiral-odd spin distribution functions coupled with a time-reversal-odd fragmentation function. This fragmentation function offers a means to measure transversity in future experiments using a transversely polarized target.

We thank M. Anselmino, J. Collins, A. M. Kotzinian, and P. J. Mulders for many interesting discussions. We gratefully acknowledge the DESY management for its sup- port, the staffs at DESY and the collaborating institutions for their significant effort, and our funding agencies for financial support.

*Deceased.

[1] J. P. Ralston and P. E. Soper, Nucl. Phys. B152, 109 (1979); R. Jaffe and X. Ji, Nucl. Phys. B375, 527 (1992).

[2] J. Collins, Nucl. Phys. B396, 161 (1993).

[3] A. M. Kotzinian, Nucl. Phys. B441, 234 (1995).

[4] P. J. Mulders and R. D. Tangerman, Nucl. Phys. B461, 197 (1996).

[5] R. Jaffe, X. Jin, and J. Tang, Phys. Rev. Lett. 80, 1166 (1998).

[6] M. Anselmino, M. Boglione, and F. Murgia, Phys. Lett. B 362, 164 (1995).

[7] D. Adams et al., Phys. Lett. B 264, 462 (1991); A. Bravar et al., Phys. Rev. Lett. 77, 2626 (1996); B. E. Bonner et al., Phys. Rev. D 41, 13 (1990).

[8] G. Gasiorowicz, Elementary Particle Physics (Wiley, New York, 1966).

[9] A. V. Efremov, O. G. Smirnova, and L. G. Tkachev, Nucl. Phys. B. (Proc. Suppl.) 74, 49 (1999).

[10] F. Stock et al., Nucl. Instrum. Methods Phys. Res., Sect. A 343, 334 (1994).

[11] D. P. Barber et al., Phys. Lett. B 343, 436 (1995).

[12] K. Ackerstaff et al., Nucl. Instrum. Methods Phys. Res., Sect. A 417, 230 (1998).

[13] J. Levelt and P. J. Mulders, Phys. Lett. B 338, 357 (1994).

[14] K. Hagiwara, K. Hikasa, and N. Kai, Phys. Rev. D 27, 84 (1983); T. Gehrmann, Report No. DTP/96/84 and hep-ph/ 9608469; K. A. Oganessyan, hep-ph/9806420.

[15] A. Schäfer and O. Teryaev, hep-ph/9908412.

[16] P. Mulders and M. Boglione, Nucl. Phys. A666-667, 257 (2000); A. M. Kotzinian et al., Nucl. Phys. A666-667, 290 (2000).

[17] A. Brandenburg, D. Muller, and O. Teryaev, Phys. Rev. D 53, 6180 (1996); A. Schäfer, and O. Teryaev (unpublished).

[18] X. Artru, J. Czyzewski, and H. Yabuki, Z. Phys. C 73, 527 (1997).

[19] M. Anselmino, M. Boglione, and F. Murgia, hep-ph/ 9810228.

[20] K. A. Oganessyan, H. Avakian, N. Bianchi, and A.M. Kotzinian, hep-ph/9808368.

[21] I. Akushevich, N. Shumeiko, and A. Soroko, hep-ph/ 9903325. 